\title{
Genome-wide (ChIP-seq) identification of target genes regulated by WRKY33 during submergence stress in Arabidopsis
}

\author{
Junlin Zhang ${ }^{\dagger}$, Bao Liư ${ }^{\dagger}$ Yan Song, Yang Chen, Jiao Fu, Jianquan Liu, Tao Ma, Zhenxiang Xi and Huanhuan Liu*
}

\begin{abstract}
Background: Hypoxia induced by flooding causes significant losses to crop production almost every year. However, the molecular network of submergence signaling pathway is still poorly understood. According to previous studies, transgenic plants overexpressing the WRKY33 gene showed enhanced resistance to submergence stress. Thus, this transcription factor may regulate a series of target genes in response to submergence. Here, to determine putative downstream targets of WRKY33 at a genome-wide scale in Arabidopsis thaliana, we performed the chromatin immunoprecipitation sequencing (ChIP-seq) using 35S:FLAG-WRKY33 overexpression transgenic lines (WRKY33-OE) after $24 \mathrm{~h}$ of submergence treatment.
\end{abstract}

Results: Using ChIP-seq data, we identified a total of 104 WRKY33-binding genes under submergence stress (WRKY33BGSs). Most WRKY33BGSs are involved in the oxidation-reduction process, programmed cell death in response to reactive oxygen species, lipid biosynthesis process, and other processes related to stress responses. Moreover, the major motif identified in the WRKY33BGSs promoters is a new cis-element, TCTCTC (named here as "TC box"). This cis-element differs from the previously known W box for WRKY33. Further qPCR experiments verified that genes carrying this motif in their promoters could be regulated by WRKY33 upon submergence treatment.

Conclusions: Our study has identified a new putative binding motif of WRKY33 and recovered numerous previously unknown target genes of WRKY33 during submergence stress. The WRKY33 gene positively participates in flooding response probably by transcriptional regulation of the downstream submergence-related target genes via a "TC box".

Keywords: WRKY33, Submergence treatment, Hypoxia, ChIP-seq, Arabidopsis

\section{Background}

Large areas of cropland in the world are subject to seasonal flooding, which causes significant losses to crop production almost every year. The diffusion of oxygen in water is 10 , 000 times slower than that in air [1], drastically reducing the supply of oxygen to the plants. Morphological adaptations of plants to low-oxygen stress include the formation

\footnotetext{
* Correspondence: liuhuanhuan85@163.com

${ }^{\dagger} J$ Junlin Zhang and Bao Liu contributed equally to this work.

Key Laboratory for Bio-resources and Eco-environment \& State Key Lab of Hydraulics \& Mountain River Engineering, College of Life Science, Sichuan University, Chengdu 610065, China
}

of adventitious roots, as well as the development of cortical air spaces in roots that promote air transport [2]. Meanwhile, the induction of fermentation pathway enzymes has been established as an important metabolic adaptation to anaerobiosis [3, 4]. Over the last decade, it has become increasingly evident that the N-degron pathway plays a wellcharacterized role in the response to hypoxia through flooding and plant submergence [5, 6]. In addition, a variety of transcription factors (TFs) have been reported to regulate gene expression that promotes adaptive responses to the environmental and physiological stress [7], including the

(c) The Author(s). 2021 Open Access This article is licensed under a Creative Commons Attribution 4.0 International License, which permits use, sharing, adaptation, distribution and reproduction in any medium or format, as long as you give appropriate credit to the original author(s) and the source, provide a link to the Creative Commons licence, and indicate if changes were made. The images or other third party material in this article are included in the article's Creative Commons licence, unless indicated otherwise in a credit line to the material. If material is not included in the article's Creative Commons licence and your intended use is not permitted by statutory regulation or exceeds the permitted use, you will need to obtain permission directly from the copyright holder. To view a copy of this licence, visit http://creativecommons.org/licenses/by/4.0/ The Creative Commons Public Domain Dedication waiver (http://creativecommons.org/publicdomain/zero/1.0/) applies to the data made available in this article, unless otherwise stated in a credit line to the data. 
Dof (DNA-binding with one finger) gene family [8], the MADS-box gene family [9], and the WRKY gene family.

The WRKY TF family, found exclusively in green plants, is characterized by the highly conserved amino acid sequence WRKYGQK at the N-terminus and the zinc-finger structure at the C-terminus [10]. Numerous studies have demonstrated that WRKY TFs are involved in regulation of various processes, such as seed germination, leaf senescence, and the responses to biotic and abiotic stresses [11, 12]. In particular, one member of the WRKY TF family, WRKY33, has been shown to regulate plant defense responses to a variety of stresses $[13,14]$. For example, previous studies have documented that overexpression of the WRKY33 gene enhances the resistance to oxidative stress [15] and promotes pathogen defense [16]. In addition, our recent study found that overexpression of WRKY33 can enhance the submergence tolerance of Arabidopsis mainly via directly up-regulating the gene RAP2.2 [17]. We further revealed that WRKY33 together with WRKY12 in up-regulating RAP2.2 expression during submergence response, meanwhile WRKY33 level is increased in RAP2.2overexpressing plants and further experiments confirmed a positive feedback regulation of WRKY33 by RAP2.2 during submergence response in Arabidopsis thaliana [17]. It has been shown that WRKY33 acts as a key factor in submergence response of Arabidopsis thaliana, however the downstream regulatory network governed by WRKY33 is still poorly understood. In this work, we used ChIP-seq to identify all WRKY33-targeted genes in response to submergence, which will provide a more clearly regulation pathway mediated by WRKY33.

\section{Results}

Verification of the function and phenotype of 35S:FLAGWRKY 33 transgenic Arabidopsis in submergence response A previous study showed that WRKY33 was induced by hypoxia stress in roots of Arabidopsis [4]. Recently, WRKY33 was reported to positively regulate submergence response via interacting with WRKY12 to directly upregulate RAP2.2 in Arabidopsis [17]. To further identify other WRKY33 targeted genes during submergence response at a genome-wide scale, we use 35S:FLAG-WRKY33 overexpression transgenic plants (WRKY33-OE) upon 24h' submergence treatment for ChIP-seq. Before the ChIP experiment, we obtained the WRKY33OE transgenic plants (Supplemental Fig. 1) in Col background and examined its submergence tolerance to make sure that the plants were workable. The phenotypic assay showed that WRKY33OE plants were more tolerant to submergence treatment compared to Col (Supplemental Fig. 2A). Survival rates and dry weights of Col, WRKY33OE-1 and WRKY33OE-2 plants were also consistent with their phenotypic assays (Supplemental Fig. 2B-C). Malondialdehyde (MDA) contents (Supplemental Fig. 2D) were also evaluated among Col, WRKY33OE-1 and WRKY33OE-2 plants and the results also supported that overexpression of FLAG-WRKY33 enhanced the submergence tolerance in Arabidopsis. Compared to wild-type, the results indicate that WRKY33-OE transgenic plants could be used to identify downstream targets of WRKY33 via ChIP-seq.

\section{Analysis of the ChIP-seq peaks}

Having confirmed that the WRKY33OE transgenic plants had the enhanced submergence resistance, we then performed the ChIP experiment firstly by using the samples ( $2 \mathrm{~g}$ pooled leaf materials) of 14-day-old seedlings of WRKY33OE1 and WRKY33OE2 plants after submergence treatment for $24 \mathrm{~h}$. The average size of the input fragments and the anti-FLAG ChIP libraries were approximately 100-400 bp. The immunoprecipitated DNA fragments were then sent to the BGI (Shenzhen, China) company for further sequencing. The input library had 25.4 million reads and the FLAG Ab ChIP library had 24.6 million reads. More than $95 \%$ of the reads were mapped to the Arabidopsis genome. The MACS2 program (Analysis based on ChIP-seq models) [18] was used to identify the enriched regions using a false discovery cutoff of 0.05 . The location of the enriched peaks in the Arabidopsis genome is shown in the supplemental Table 1 (Additional file 3). Of the 393 enriched regions, $24 \%$ of the peaks were in genetic regions (from $2 \mathrm{~kb}$ upstream of the start of transcription to $2 \mathrm{~kb}$ downstream of the stop codon, including the coding region). Of the peaks that were in the genetic regions, $22 \%$ located only in the promoter regions, $48 \%$ in the promoter and exons or introns regions, only $26 \%$ in exons and introns (Fig. 1). After calling peak, we aimed to examine the peak locations among the whole genome. We then used the covplot function in ChIPseeker (an R package for ChIP peak Annotation, Comparison and Visualization) to calculate the coverage of peak regions over the chromosomes. We generated a figure for visualization (Fig. 2a). Since some annotations overlapped, we then viewed the complete annotations with overlap through the vennpie function in ChIPseeker (Fig. 2b). Table 1 lists the genes related to the peaks in the gene region. These peaks are enriched by more than 5 fold and all have known putative functions.

\section{Motif analysis of WKRY33 TF targeted genes}

We analyzed all the promoter-located peak sequences from the ChIP-seq using MEME-ChIP [19] to identify the enriched motif, and detected the two types of motifs (Fig. 3a). The most significantly enriched MEME motif is "TCTCTCTC" (E-value of 6.3e-005) which is different from the "W box" bound by WRKY33 TF reported previously. We then named it as "TC box" (Fig. 3b). The next most significant motif is AAAAWAAA (E-value of $3.1 \mathrm{e}+002$ ) (Fig. 3c). WRKY proteins can repress or 


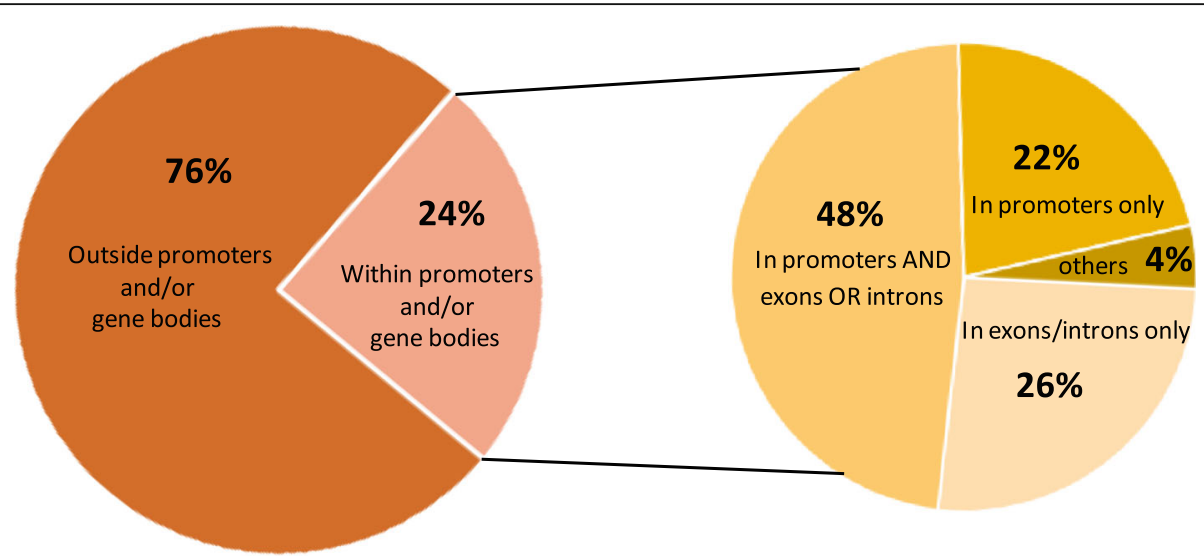

Fig. 1 Distribution of ChIP peaks in the genome. Percentage of peaks that reside $2 \mathrm{~kb}$ upstream of the transcription start site or $2 \mathrm{~kb}$ downstream of the stop codon (gene body), and location of the peaks in the gene bodies

activate the expression of downstream genes via binding to the W-box (TGACC $(\mathrm{A} / \mathrm{T}))$ in promoter of its target genes upon pathogen defense [18]. The identified "TC box" motif may responsible for the activation or repression of submergence-related target genes which still needs further verifications.

\section{Gene ontology analysis to identify biological and \\ functional enriched categories}

Gene Ontology (GO) analyses using the Enrich GO [20] revealed $61 \mathrm{GO}$ categories belonging to the Biological Process (BP) ontology, which were determined to be significantly over-represented in the ChIP-seq sample relative to the Arabidopsis genome (fisher <0.01, Additional file 4). The top 10 significantly enriched GO biological processes of WRKY33BGSs were shown in Fig. 4a. The results of the top 20 extremely significant enrichments (Fig. 4b) suggest that the gene ontology related to the submergence response includes the oxidation-reduction process, programmed cell death in response to reactive oxygen species and lipid biosynthesis process. Additional biological processes including cellular response to auxin stimulus, response to hydrogen peroxide were also identified when using a fisher greater than 0.01 and less than 0.05 (Additional file 4). Plant phytohormones, such as auxin, may also participate in the submergence response process as suggested by our Gene Ontology (GO) analysis, which still needs further experimental validation.

\section{Expression analysis of genes contain the "TC box" in Col} and WRKY33OE plants after submergence treatment

WRKY33 may regulate its downstream target genes directly via the identified "TC box" during submergence response. To further validate this hypothesis, we selected four genes that contain the "TC box" and performed a qPCR test. The results showed the expression levels of these four genes were all regulated by WRKY33 transcription factor. At2G35736 gene was downregulated by WRKY33 while the other three genes At1G66810,
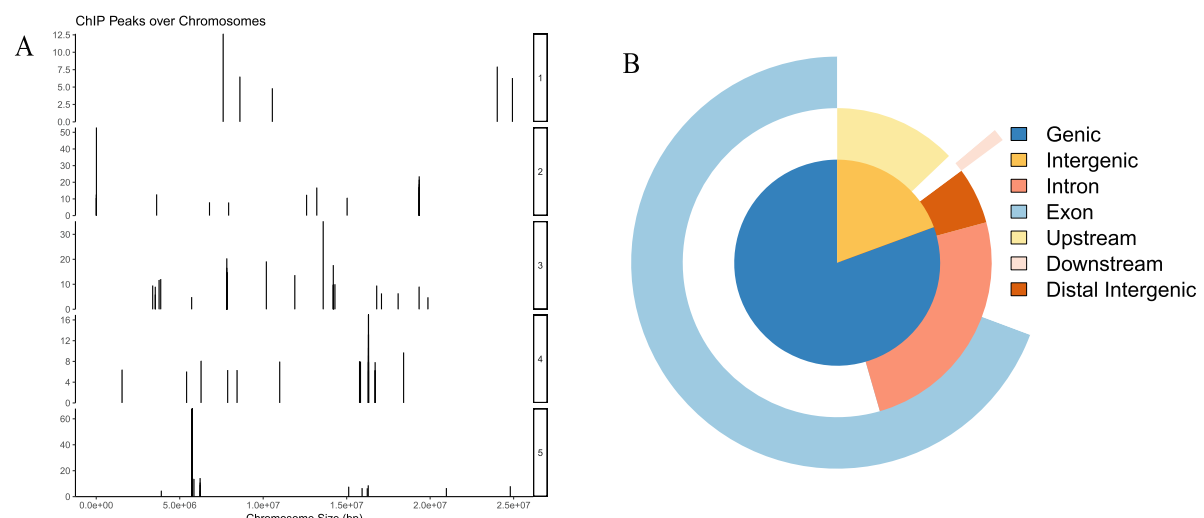

Fig. 2 The location of all ChIP peaks over chromosome. a ChIP peaks coverage plot: the right ordinate represents the chromosome, the left ordinate represents the size of the peak, and the abscissa represents the size of the chromosome. $\mathbf{b}$ Genomic Annotation by vennpie. Visually shows the full annotation with their overlap 
Table 1 List of genes and their putative function

\begin{tabular}{|c|c|c|}
\hline Gene Name & Putative Function & Fold-Change \\
\hline AT1G21650 & Preprotein translocase SecA family protein & 8.0 \\
\hline AT1G64628 & conserved peptide upstream open reading frame 57 & 5.2 \\
\hline AT2G01008 & maternal effect embryo arrest protein & 8.1 \\
\hline AT2G15540 & non-LTR retrotransposon family & 5.2 \\
\hline AT2G18220 & Noc2p family & 5.2 \\
\hline AT2G29350 & senescence-associated gene 13 & 7.7 \\
\hline AT2G31040 & Encodes an integral thylakoid protein that facilitates assembly of the membranous part of the chloroplast ATPase & 9.6 \\
\hline AT2G47090 & zinc ion binding/nucleic acid binding protein & 12.4 \\
\hline AT3G10810 & zinc finger (C3HC4-type RING finger) family protein & 6.4 \\
\hline AT3G11280 & Duplicated homeodomain-like superfamily protein & 5.9 \\
\hline AT3G11900 & aromatic and neutral transporter 1 & 7.1 \\
\hline AT3G12120 & fatty acid desaturase 2 & 7.8 \\
\hline AT3G22160 & JAV1 is a repressor of jasmonate-mediated defense responses & 11.3 \\
\hline AT3G22170 & far-red elongated hypocotyls 3 & 9.5 \\
\hline AT3G27503 & Encodes a member of a family of small, secreted, cysteine rich proteins with sequence similarity to SCR & 10.6 \\
\hline AT3G30250 & transposable element gene & 8.0 \\
\hline AT3G33058 & gypsy-like retrotransposon family & 15.7 \\
\hline AT3G41768 & rRNA & 10.2 \\
\hline AT3G41979 & 5.8SrRNA & 6.8 \\
\hline AT3G42130 & glycine-rich protein & 6.2 \\
\hline AT3G45755 & transposable element gene & 6.1 \\
\hline AT3G52140 & tetratricopeptide repeat (TPR)-containing protein & 6.2 \\
\hline AT4G10030 & Alpha/beta hydrolase domain containing protein involved in lipid biosynthesis & 5.3 \\
\hline AT4G20360 & Nuclear transcribed, plastid localized EF-Tu translation elongation factor & 5.2 \\
\hline AT4G32700 & helicases;ATP-dependent helicases;nucleic acid binding;ATP binding;DNA-directed DNA polymerases;DNA binding & 5.3 \\
\hline AT4G32810 & carotenoid cleavage dioxygenase 8 & 5.2 \\
\hline AT4G34035 & pre-tRNA tRNA-Arg & 9.6 \\
\hline AT4G34040 & RING/U-box superfamily protein & 7.9 \\
\hline AT4G35090 & catalase 2 & 5.2 \\
\hline AT4G39672 & pre-tRNA & 6.1 \\
\hline AT5G17420 & Encodes a xylem-specific cellulose synthase that is phosphorylated on one or more serine residues & 30.1 \\
\hline AT5G17730 & P-loop containing nucleoside triphosphate hydrolases superfamily protein & 8.0 \\
\hline AT5G18650 & CHY-type/CTCHY-type/RING-type Zinc finger protein & 8.5 \\
\hline AT5G37960 & GroES-like family protein & 5.4 \\
\hline AT5G40690 & histone-lysine $\mathrm{N}$-methyltransferase trithorax-like protein & 6.1 \\
\hline AT5G61710 & cotton fiber protein & 5.3 \\
\hline
\end{tabular}

The genes listed in this table are limited to those associated with peaks that were enriched greater than 5 -fold and have been classified with a known function

At2G47090, and At3g12120 were upregulated by WRKY33 (Fig. 5). These results support that these four genes targeted by WRKY33 may participate in submergence response via the "TC box". However, further experimental validations including EMSA (electrophoretic mobility shift assay) are needed in the future to fully validate the direct regulation role of WRKY33.

\section{Discussion}

Flooding stress, one of the most important abiotic stresses, has attracted the attention of scientists over the world [21]. Many studies have revealed the molecular mechanisms of plants in response to flooding [21]. A few genes from the WRKY transcription factor family have been shown to play an important role in submergence response, including, WRKY22 [22] and WRKY33 


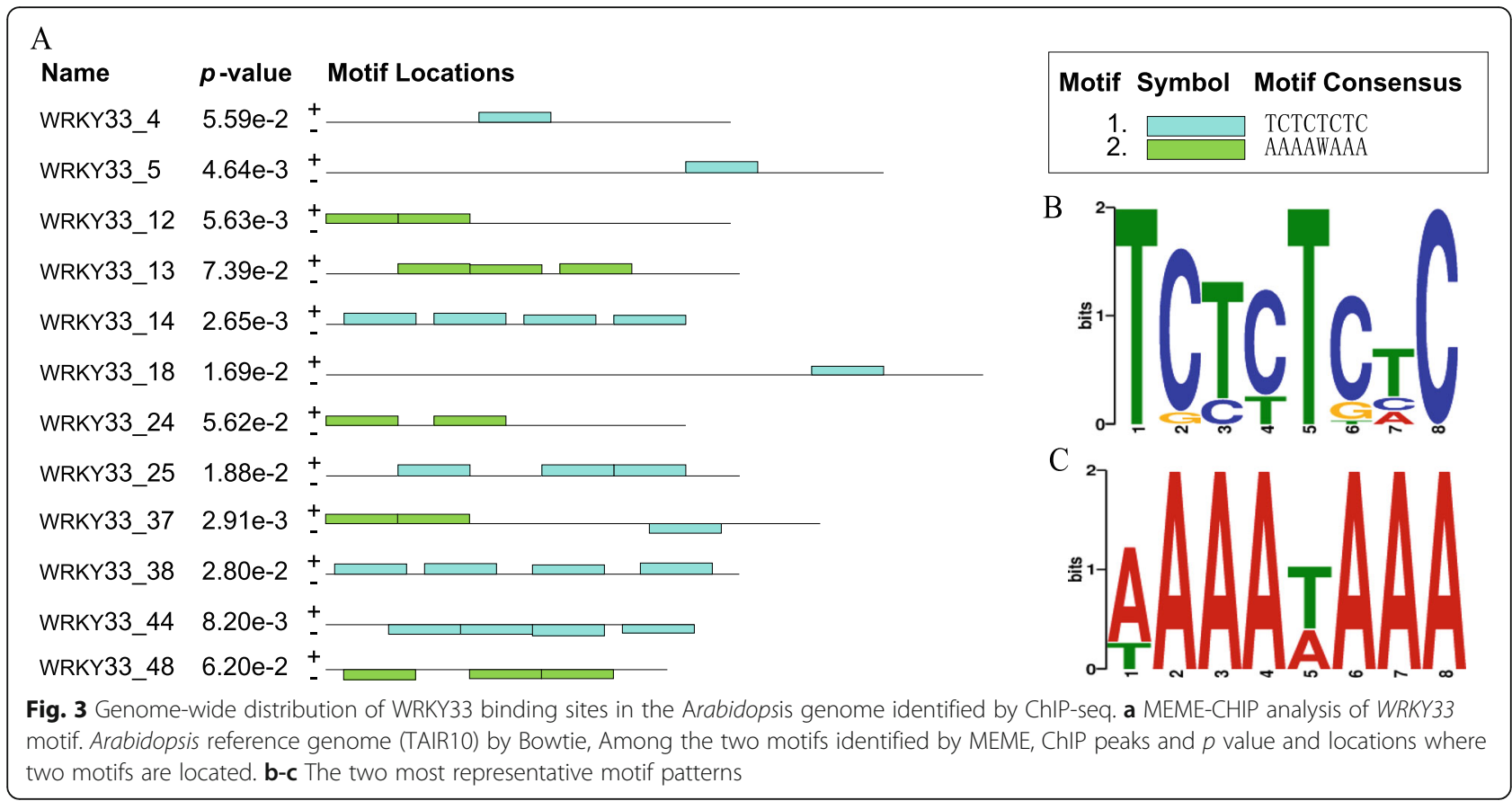

[17]. WRKY22-mediated pathways in response to submergence have shown to regulate multiple transcription factors, including WRKY29 and WRKY53 [22]. The WRKY33/WRKY12-RAP2.2 feedforward cycle in submergence response we discovered recently has confirmed the key positive role of WRKY33 in flooding response [17]. In this work, we went a step further and tried to explore the regulation network of WRKY33 during submergence stress. By phenotypic analysis, we found that plants overexpressing FLAG-WRKY33 did enhance the resistance to submergence stress compared with Col (Supplemental Fig. 2). We then used 35S: FLAG-WRKY33 overexpressing transgenic lines (WRKY33-OE) upon submergence treatment for ChIPseq, to identify the WRKY33 TF target genes at a genome-wide scale. By ChIP-seq analyses, we identified 104 WRKY33-binding genes upon submergence stress (WRKY33BGSs) and gene enrichment analysis showed that these genes participate in oxidoreductase reactions, lipid biosynthetic process and other functions. Most of these identified genes are reported for the first time for submergence stress. The major motif that we identified in the WRKY33BGSs promoters is the "TC box" ciselement. This candidate motif for WRKY33 TF may regulate genes expression during submergence stress. Our further functional analyses of all identified genes suggest that WRKY33BGSs may protect cells from oxidative stress and other processes to improve the tolerance ability upon submergence stress.

The identified "TC box" cis-element is a new motif different from the known "W box" element for WRKY33 and may be specific to regulating the target genes during submergence stress. WRKY33 can regulate RAP2.2 expression via the $\mathrm{W}$ box element only during the submergence response [17]. Interestingly, there also is a "TC box" sequence "TCTCTC" in the promoter region $(-1$, $875 \mathrm{bp)}$ of $R A P 2.2$. Previous studies have shown that the TFs have different binding abilities towards different ciselements upon different conditions. For example, IPA1 was reported to bind to the "GTAC" element in the promoter of DEP1 in the normal condition while bind to the "TGGGCC" element in the promoter of WRKY45 upon pathogen infection [23]. This switch is mediated by the phosphorylation of IPA1 protein. Submergence treatment might also induce the phosphorylation of WRKY33 like IPA1 upon pathogen infection [17, 21]. In addition, this TF may also have different binding abilities towards "W box" or "TC box" elements between normal growth and submergence treatment conditions like IPA1. Such a difference in binding ability may be mediated by the protein post-transcriptional modifications of WRKY33.

In this study, we obtained a more comprehensive understanding of the submergence stress response mediated by WRKY33. The ChIP-seq candidate genes regulated by WRKY33 provide a more comprehensive understanding of the molecular basis of plant submergence response. These genes can be further manipulated to improve stress tolerances when their functions and regulation pathways are well clarified. In addition, the functions of genes induced by low-oxygen stress seem to overlap those induced by other biotic or abiotic stress 


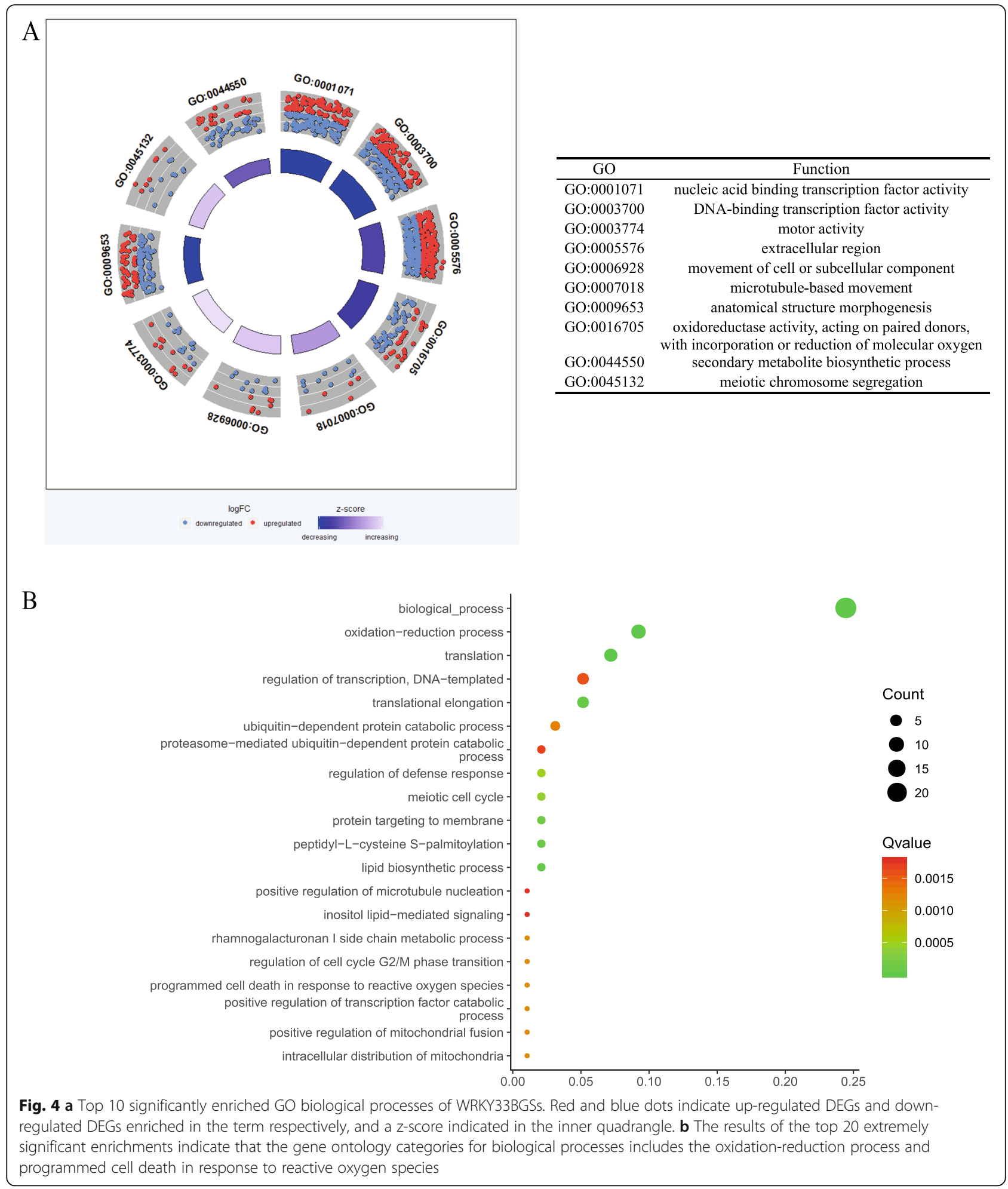

responses [24]. It is worth noting that only roles of WRKY33 in leaves during submergence response were examined here. However, its function may be altered by using different tissues, since WRKY33 also is highly expressed in roots [25]. The hypoxic response including many physiology processes, such as aerobic metabolism, carbon and energy partition, redox balance, ethylene accumulation, gene regulation cascades [26] and so on, is complex. The work we have done is just the tip of the iceberg and more works are still needed to clarify the 
A

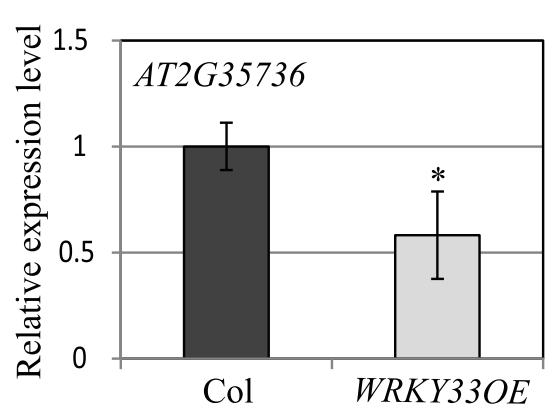

$\mathrm{C}$

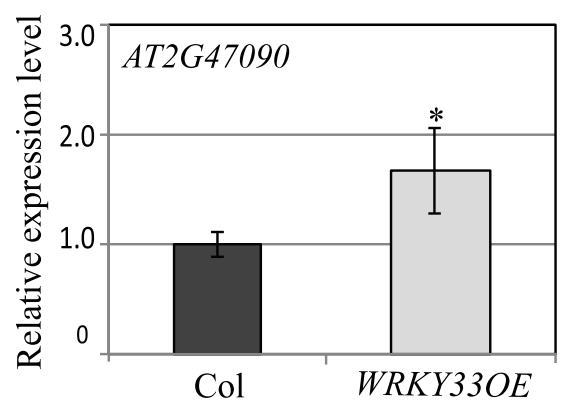

B

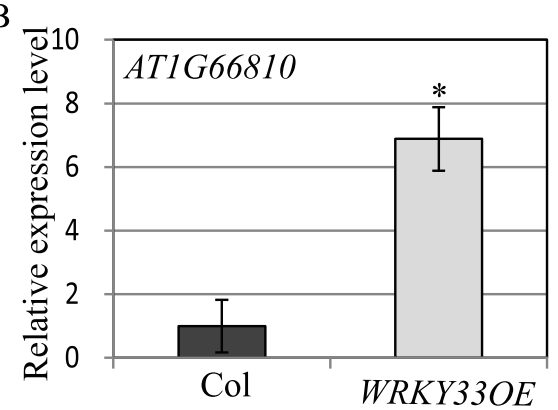

$\mathrm{D}$

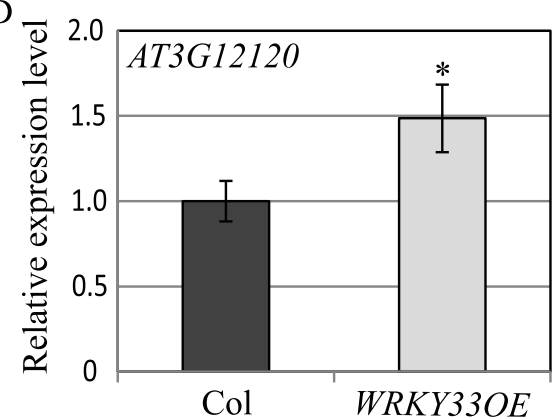

Fig. 5 Expression analysis of genes containing the "TC box" in Col and WRKY33OE plants after submergence treatment. a AT2G35736 gene is downregulated by WRKY33 upon submergence treatment for $24 \mathrm{~h}$. b-d AT1G66810, AT2G47090 and AT3G12120 genes are upregulated by WRKY33 upon submergence treatment for $24 \mathrm{~h}$. Three independent biological replicates were used. Data are average values \pm SD $(n=3)$ of 3 biological replicates. ${ }^{*}(p<0.05$, according to Student's $t$-test) indicates significant difference from Col

mechanism of submergence response of plants in the future.

\section{Conclusion}

We identified numerous previously unknown direct target genes of WRKY33 in response to submergence stress by ChIP-Seq and a new cis-element "TC box" was identified. Our work suggested that WRKY33 TF may positively participates in flooding response via the "TC box" to its target genes. Thus, our results provide new insights into the functions of WRKY33 transcription factor and the submergence response of Arabidopsis.

\section{Methods}

\section{Arabidopsis growing conditions and submergence} treatment

Briefly, cDNA was prepared from 4-week rosette leaves of Arabidopsis and was diluted to 50 times. The diluted cDNA was then used as a template to amplify the WRKY33, which was inserted into a vector tagged by FLAG tag, under the control of the $35 S$ promoter. The construct was transformed into Agrobacterium strain GV3101 [27], which was used to transform Arabidopsis using the floral dip method and identified by hygromycin screening followed by qRT-PCR analysis of their expression levels. The 35S:FLAG-WRKY33 (WRKY33OE) transgenic plants we used were obtained in this work.
All materials were grown at $22^{\circ} \mathrm{C}$ in a 16 - $\mathrm{h}$ light $/ 8$ - $\mathrm{h}$ dark cycle. Seeds were germinated on $1 / 2$ MS medium $(\mathrm{pH}=5.85)$ for 7 days and then transplanted into soil.

For submergence treatments, 4-week-old plants were submerged $10 \mathrm{~cm}$ below the surface of the water in darkness for $50 \mathrm{~h}$. All submergence treatments started at 9: 00 a.m. Twelve Col and WRKY33OE plants were used for submergence treatment every time. The total experiments were repeated three times.

For ChIP-sequencing, 4-week-old 35S:FLAG-WRKY33 transgenic plants were submerged $10 \mathrm{~cm}$ below the surface of the water in darkness for $24 \mathrm{~h}$. Then rosette leaves were collected for ChIP experiments. All submergence treatments started at 9:00 a.m.

\section{Malondialdehyde measurements}

The Malondialdehyde (MDA) was measured according to a previous study [28]. 4-week-old rosette leaves of 10 plants treated by dark submergence were weighed and pulverized in 5\% trichloroacetic acid buffer, and then mix the supernatant with $6.7 \%$ thiobarbituric acid and $5 \%$ trichloroacetic acid buffer. The materials were further incubated at $100{ }^{\circ} \mathrm{C}$ for $0.5 \mathrm{~h}$, and then cooled to the room temperature. The absorbance was measured at 532,450 , and $600 \mathrm{~nm}$ with a spectrophotometer plate reader. 


\section{ChIP and ChIP-sequencing}

Samples of 14-day-old seedlings of WRKY33OE1 and WRKY33OE2 plants were dark submergence treated for $24 \mathrm{~h}$ and fixed using $1 \%$ formaldehyde and prepared for chromatin immunoprecipitation assays, as previously described [29]. The DNA-protein complexes were extracted from rosette leaves ( $2 \mathrm{~g}$ pooled leaf materials) of 4-week-old 35S:FLAG-WRKY33 OE1 and OE2 transgenic plants, and pulled down using anti:FLAG antibody (Sigma-Aldrich F1084) and protein A Agarose beads following the ChIP protocol [30]. The immunoprecipitated DNA fragments were dissolved in $40 \mu \mathrm{lddH} 2 \mathrm{O}$ and then sent to the BGI (Shenzhen, China) company for the following experiment. $10 \%$ of the total DNA-protein complexes before the immunoprecipitation were used as the input DNA.

ChIP-seq service was performed by BGI company (Shenzhen, China). The DNA is combined with End Repair Mix and incubated at $20^{\circ} \mathrm{C}$ for $30 \mathrm{~min}$. We further purified the end-repaired DNA with QIAquick PCR Purification Kit (Qiagen), and added A-Tailing Mix and incubated at $37^{\circ} \mathrm{C}$ for $30 \mathrm{~min}$. We combined the purified Adenylate 3 'Ends DNA, Adapter and Ligation Mix and incubated the ligation reaction at $20^{\circ} \mathrm{C}$ for $15 \mathrm{~min}$. We purified the Adapter-ligated DNA with the QIAquick PCR Purification Kit. We conducted several rounds of PCR amplification with PCR Primer Cocktail and PCR Master Mix to enrich the Adapter-ligated DNA fragments. Then the PCR products are selected (about 100$300 \mathrm{bp}$, including adaptor sequence) by running a $2 \%$ agarose gel to recover the target fragments. We purified the gel with QIAquick Gel Extraction kit (QIAGEN). The final library was quantitated in two ways: determining the average molecule length and sample integrity and purity using the Agilent 2100 bioanalyzer instrument (Agilent DNA 1000 Reagents) and quantifying the library by real-time quantitative PCR (qPCR). The double stranded PCR products were heat-denatured and circularized by the splint oligo sequence. The single strand circle DNA (ssCir DNA) was formatted as the final library. Library was qualified by Qubit ssDNA kit. The sequencing was performed with the BGISEQ-500 sequencing system, featured by combinatorial probeanchor synthesis (cPAS) and DNA Nanoballs (DNB) technology for superior data quality (BGI-Shenzhen, China).

The raw sequencing image data were examined by the Illumina analysis pipeline. ChIP-seq reads were aligned to the Arabidopsis reference genome (TAIR10) by Bowtie [31] with at most 2 mismatches. The input group was used as a control. The results were visualized with IGV software. Reads that appeared more than twice at the same position on the same strand were discarded to remove PCR duplication. MACS2 (Model-based Analysis of ChIP-seq) [32] was used to identify peaks using a qvalue cutoff of 0.05 .

\section{Motif analysis}

To identify possible binding motif of the WRKY33 transcription factor, the ChIP peak sequences were subjected to MEME (Multiple EM for Motif Elicitation)-ChIP [19]. The MEME-ChIP program uses two ab initio motif discovery algorithms: MEME [19], and DREME (Discriminative Regular Expression Motif Elicitation) [33], which uses regular expressions to search for short eukaryotic TF motifs that are missed by MEME.

\section{Gene function of WRKY33 TF target genes}

In order to determine the putative functions of the target gene WRKY33, all identifed genes with ChIP-seq peaks in the upstream promoter region or the potential regulatory region downstream were subjected to annotation of the categories of ontological genes (GO) [20]. The default Fisher's Exact Test and Benjamini-Yekutieli multiple test correction methods [34] were used to generate $p$-values for statistical significance and corresponding False Discovery Rate (FDR) values.

\section{RNA extraction and quantification}

Total RNA was isolated using the Biospin Plant Total RNA Extraction kit according to the user manual (Bioer Technology; Hangzhou, China), from the pooled threeweek old rosette leaves of $\mathrm{Col}$ and 35S:FLAG-WRKY33 plants, and 1-2 $\mu$ g total RNA was used for reverse transcription, using the PrimeScript RT reagent kit (Takara Cat\# RR047A). A QuantiNova SYBR Green PCR Kit was used for qPCR reactions with qPCR-specific primers. The expression levels of putative target genes were compared with Arabidopsis ACTIN genes.

\section{Abbreviations}

At: Arabidopsis thaliana; ChIP: Chromatin immunoprecipitation; DREME: Discriminative Regular Expression Motif Elicitation; GO: Gene Ontology; MEME: Multiple EM for Motif Elicitation; RT: Reverse transcriptase; seq: Sequencing; TF: Transcription factor

\section{Supplementary Information}

The online version contains supplementary material available at https://doi. org/10.1186/s12863-021-00972-5.

Additional file 1: Supplemental Fig. 1. Identification of WRKY33 overexpressing transgenic plants.

Additional file 2: Supplemental Fig. 2. WRKY33 positively regulates the submergence response in Arabidopsis.

Additional file 3. List of enriched peaks and their location in the Arabidopsis genome.

Additional file 4. The putative function of the target gene WRKY33. And primers used in this study.

Additional file 5. Primers used in this study. Primers used for vector construction and gene expression analysis. 


\section{Acknowledgements}

Thanks to the supports by National Natural Science Foundation of China and the Fundamental Research Funds for the Central Universities.

\section{Authors' contributions}

LHH and ZJL designed the experiments; ZJL, LB, SY, CY and FJ performed the experiments, ZJL analyzed the data for the work; $L H H, Z J L$ and $L B$ wrote the article, XZX, LJQ and MT revised the article. LHH, ZJL and LB revised the article according to the reviewers. All authors have read and approved the final version of the manuscript.

\section{Funding}

This research was equally supported by the National Natural Science Foundation of China (31870244) and the Fundamental Research Funds for the Central Universities (grant No. SCU2019D013). The funding bodies didn't play any roles in the design of the study, interpretation of data or writing the manuscript.

\section{Availability of data and materials}

All data generated are included in this published article and its supplementary files. The raw sequence data reported in this paper have been deposited in the Genome Sequence Archive (Genomics, Proteomics \& Bioinformatics 2017) in National Genomics Data Center (Nucleic Acids Res 2021), China National Center for Bioinformation / Beijing Institute of Genomics, Chinese Academy of Sciences, under accession number CRA003775 that are publicly accessible at https://bigd.big.ac.cn/gsa.

\section{Declarations}

\section{Ethics approval and consent to participate}

The seeds of Arabidopsis thaliana we used were kept in our lab in the Key Laboratory for Bio-resources and Eco-environment, College of Life Science, Sichuan University. The experimental methods conducted in this study complied with current Chinese laws and regulations. The trade name, company name, or company name used in this publication is to provide readers with information and convenience. Such use does not constitute an official endorsement or endorsement of any product or service by the Ministry of Agriculture or Agricultural Research Service Department of China, does not exclude other suitable products or services.

\section{Consent for publication}

Not applicable.

\section{Competing interests}

The authors declare that they have no competing interests.

Received: 27 November 2020 Accepted: 17 May 2021

Published online: 24 May 2021

\section{References}

1. Armstrong W. Aeration in higher plants. Adv Bot Res. 1979;7:225-332.

2. Visser EJW, Voesenek LACJ, Vartapetian BB, Jackson MB. Flooding and plant growth. Academic Press; 1984. https://doi.org/10.1016/B978-0-12-424120-6. 50009-2. https://doi.org/10.1016/C2009-0-02985-7.

3. Kennedy RA, Rumpho ME, Fox TC. Anaerobic metabolism in plants. Plant Physiol. 1992;100(1):1-6. https://doi.org/10.1104/pp.100.1.1.

4. Klok EJ, Wilson IW, Wilson D, Chapman SC, Ewing RM, Somerville SC, et al. Expression profile analysis of the low-oxygen response in Arabidopsis root cultures. Plant Cell. 2002;14(10):2481-94. https://doi.org/10.1105/tpc.004747.

5. Licausi F, Kosmacz M, Weits DA, Giuntoli B, Giorgi FM, Voesenek LACJ, et al. Oxygen sensing in plants is mediated by an $\mathrm{N}$-end rule pathway for protein destabilization. Nature. 2011;479(7373):419-22. https://doi.org/10.1038/na ture10536.

6. Dissmeyer N. Conditional protein function via N-Degron pathway-mediated proteostasis in stress physiology. Annu Rev Plant Biol. 2019;70(1):83-117. https://doi.org/10.1146/annurev-arplant-050718-095937.

7. Cheatle Jarvela AM, Hinman VF. Evolution of transcription factor function as a mechanism for changing metazoan developmental gene regulatory networks. Evodevo. 2015;6(1):1-11.

8. Moreno-Risueno MÁ, Martínez M, Vicente-Carbajosa J, Carbonero P. The family of DOF transcription factors: from green unicellular algae to vascular plants. Mol Gen Genomics. 2007;277(4):379-90. https://doi.org/10.1007/ s00438-006-0186-9.

9. Martinez-Castilla LP, Alvarez-Buylla ER. Adaptive evolution in the Arabidopsis MADS-box gene family inferred from its complete resolved phylogeny. Proc Natl Acad U S A. 2003;100(23):13407-12. https://doi.org/10.1073/pnas.183 5864100 .

10. Eulgem T, Rushton PJ, Robatzek S, Somssich IE. The WRKY superfamily of plant transcription factors. Trends Plant Sci. 2000;5(5):199-206. https://doi. org/10.1016/S1360-1385(00)01600-9.

11. Rushton PJ, Somssich IE, Ringler P, Shen QJ. WRKY transcription factors. Trends Plant Sci. 2010;15(5):247-58. https://doi.org/10.1016/j.tplants.2010.02. 006.

12. Bakshi M, Oelmüller R. WRKY transcription factors: Jack of many trades in plants. Plant Signal Behav. 2014;9(2):e27700. https://doi.org/10.4161/psb.2 7700.

13. Krishnamurthy P, Vishal B, Wan JH, Lok FCJ, Kumar PP. Regulation of CYP94B1 by WRKY33 controls apoplastic barrier formation in the roots leading to salt tolerance. Plant Physiol. 2020;184(4):2199-215. https://doi. org/10.1104/pp.20.01054

14. Rajappa S, Krishnamurthy P, Kumar P. Regulation of AtKUP2 expression by bHLH and WRKY transcription factors helps to confer increased salt tolerance to Arabidopsis thaliana plants. Front Plant Sci. 2020;11:1311. https://doi.org/10.3389/fpls.2020.01311.

15. Sun Y, Liu Z, Guo J, Zhu Z, Sun X. WRKY33-PIF4 loop is required for the regulation of $\mathrm{H} 2 \mathrm{O} 2$ homeostasis. Biochem Biophys Res Commun. 2020; 527(4):922-8. https://doi.org/10.1016/j.bbrc.2020.05.041.

16. Barco B, Kim Y, Clay N. Expansion of a core regulon by transposable elements promotes Arabidopsis chemical diversity and pathogen defense. Nat Commun. 2019;10(1):3444. https://doi.org/10.1038/s41467-019-11406-3.

17. Tang $H$, Bi H, Liu B, Lou S, Yan S, Tong S, et al. WRKY 33 interacts with WRKY12 protein to up-regulate RAP2.2 during submergence induced hypoxia response in Arabidopsis thaliana. New Phytologist. 2021;229:106-25.

18. Zhang Y, Liu T, Meyer CA, Eeckhoute J, Johnson DS, Bernstein BE, et al. Model-based analysis of ChIP-Seq (MACS). Genome Biol. 2008;9(9):R137. https://doi.org/10.1186/gb-2008-9-9-r137.

19. Machanick P, Bailey T. MEME-ChIP: motif analysis of large DNA datasets. Bioinformatics. 2011;27(12):1696-7. https://doi.org/10.1093/bioinformatics/ btr189.

20. BeiBbarth T, Speed T. GOstat: find statistically overrepresented gene ontologies within a group of genes. Bioinformatics. 2004;20(9):1464-5. https://doi.org/10.1093/bioinformatics/bth088.

21. Perata $P$, Voesenek LACJ. Submergence tolerance in rice requires Sub1 $A$, an ethylene-response-factor-like gene. Trends Plant Sci. 2007;12(2):43-6. https:/ doi.org/10.1016/j.tplants.2006.12.005.

22. Hsu FC, Chou MY, Chou SJ, Li YR, Shih MC. Submergence confers immunity mediated by the WRKY22 transcription factor in Arabidopsis. Plant Cell. 2013;25(7):2699-713. https://doi.org/10.1105/tpc.113.114447.

23. Wang J, Zhou L, Shi H, Chern M, Yu H, Yi H, et al. A single transcription factor promotes both yield and immunity in rice. Science. 2018;361 (6406): 1026-8. https://doi.org/10.1126/science.aat7675.

24. Chen W, Provart NJ, Glazebrook J, Katagiri F, Chang HS, Eulgem T, et al. Expression profile matrix of Arabidopsis transcription factor genes suggests their putative functions in response to environmental stresses. Plant Cell. 2002;14(3):559-74. https://doi.org/10.1105/tpc.010410.

25. Jiang $Y$, Deyholos MK. Functional characterization of Arabidopsis $\mathrm{NaCl}$ inducible WRKY25 and WRKY33 transcription factors in abiotic stresses. Plant Mol Biol. 2009;69(1):91-105. https://doi.org/10.1007/s11103-008-9408-3.

26. Trevaskis B, Watts RA, Andersson CR, Llewellyn DJ, Hargrove MS, Olson JS, et al. Two hemoglobin genes in Arabidopsis thaliana: the evolutionary origins of leghemoglobins. Proc Natl Acad Sci U S A. 1997;94(22):12230-4. https://doi.org/10.1073/pnas.94.22.12230.

27. Zhang X, Henriques R, Lin SS, Niu QW, Chua NH. Agrobacterium-mediated transformation of Arabidopsis thaliana using the floral dip method. Nat Protoc. 2006;1 (2):641-6. https://doi.org/10.1038/nprot.2006.97.

28. Yuan $L$, Dai Y, Xie L, Yu L, Zhou Y, Lai Y, et al. Jasmonate regulates plant responses to reoxygenation through activation of antioxidant synthesis. Plant Physiol. 2017;173(3):1864-80. https://doi.org/10.1104/pp.16.01803.

29. Martin RC, Vining K, Dombrowski JE. Genome-wide (ChIP-seq) identification of target genes regulated by BdbZIP10 during paraquat-induced oxidative stress. BMC Plant Biol. 2018;18(1):58. https://doi.org/10.1186/s12870-018-12 75-8. 
30. Bowler C, Benvenuto G, Laflamme P, Molino D, Probst AV, Tariq M, et al. Chromatin techniques for plant cells. Plant J. 2010;39(5):776-89.

31. Langmead B, Trapnell C, Pop M, Salzberg SL. Ultrafast and memory-efficient alignment of short DNA sequences to the human genome. Genome Biol. 2009;10(3):R25. https://doi.org/10.1186/gb-2009-10-3-r25.

32. Feng J, Liu T, Zhang Y. Using MACS to identify peaks from ChIP-Seq data. Curr Protoc Bioinformatics. 2011;Chapter 2(1):Unit 2.14.

33. Bailey TL. DREME: motif discovery in transcription factor ChIP-seq data. Bioinformatics. 2011;27(12):1653-9. https://doi.org/10.1093/bioinformatics/ btr261.

34. Benjamini Y, Yekutieli D. The control of the false discovery rate in multiple testing under dependency. Ann Stat. 2001;29:1165-88.

\section{Publisher's Note}

Springer Nature remains neutral with regard to jurisdictional claims in published maps and institutional affiliations.

Ready to submit your research? Choose BMC and benefit from:

- fast, convenient online submission

- thorough peer review by experienced researchers in your field

- rapid publication on acceptance

- support for research data, including large and complex data types

- gold Open Access which fosters wider collaboration and increased citations

- maximum visibility for your research: over $100 \mathrm{M}$ website views per year

At $\mathrm{BMC}$, research is always in progress.

Learn more biomedcentral.com/submissions 